\title{
Spacetime Locality of the BRST Formalism
}

\section{Marc Henneaux ${ }^{\star}$}

Faculté des Sciences, Université Libre de Bruxelles, Campus Plaine C.P. 231, B-1050 Bruxelles, Belgium and Centro de Estudios Cientificos de Santiago, Casilla 16443, Santiago 9, Chile

Received May 21, 1990; in revised form December 10, 1990

\begin{abstract}
The spacetime locality of the BRST formalism is investigated. The analysis covers gauge theories with either closed or open algebras and is undertaken in the explicit context of the antifield formulation of the BRST theory. Under appropriate conditions, the homology of the Koszul-Tate differential modulo the spacetime exterior derivative is shown to be trivial in the space of non-integrated densities with positive antighost and pure ghost numbers. As a result: (i) the solution of the master equation can be taken to be a local functional; (ii) the gauge fixed action is also a local functional provided one takes the gauge fixing fermion to be a local functional as well; and (iii) the BRST transformation is local.
\end{abstract}

\section{Introduction}

It has become clear in the last years that the BRST symmetry provides an extremely powerful tool for studying the geometrical, algebraic and quantum properties of gauge theories. However, as pointed out by many authors, it is not completely obvious that the BRST construction preserves spacetime locality of the field theoretical formalism. For this reason, a "locality hypothesis" has been formulated, expressing that the BRST symmetry is local is spacetime.

The purpose of the paper is to show that the hypothesis on the locality of the BRST formalism is actually superfluous. More precisely, it is proved that under appropriate conditions, (i) the BRST transformation is local; and (ii) the gauge fixed action appearing in the path integral is a local functional. The spacetime locality of the gauge fixed action enables one to describe the theory in terms of fundamental local interaction processes. It appears to be important for the perturbative quantum properties and the renormalization of the theory. Locality also plays a key role in the proof of important theorems of quantum field theory (PCT theorem, etc...).

* Maître de recherches au Fonds National de la Recherche Scientifique (Belgium) 
The analysis given below covers gauge theories with either closed or open algebras. This analysis is undertaken in the explicit context of the antifield formulation of the BRST theory $[1,2]$. It can be carried out along the same lines in the Hamiltonian formulation $[3,4]$ to prove spatial locality of the BRST charge.

For the sake of avoiding cumbersome notations, only irreducible bosonic gauge theories are considered. The results are easily extended to the general case of a reducible theory with both bosonic and fermionic gauge symmetries.

\section{Is the Koszul-Tate Differential Acyclic in the Space of Local Functionals?}

The question of locality of the gauge-fixed action was first raised in [5] and arises as follows. The action $S_{0}\left[\phi^{i}\right]$ of a gauge system cannot be used as such in the path integral. It needs to be "gauge fixed." In the case of an open gauge algebra, or when the gauge transformations are reducible on-shell, the Faddeev-Popov algorithm cannot be applied, and the gauge fixed action contains generically quartic (or even higher order) ghost interactions. The most expedient way for deriving the correct gauge fixed action is based on the antifield formalism, where one introduces one antifield for each field or ghost in the theory. The antifields are conjugate to the corresponding fields (or ghosts) in the so-called "antibracket," which is denoted by $($, ). The central equation of the formalism is the master equation $(S, S)=0$. The gauge fixed action is obtained by setting in $S$ the antifields equal to the functional derivatives of an appropriate "gauge fixing fermion" $\psi$ with respect to the conjugate variables. Different choices of $\psi$ correspond to different gauges and yield the same physical amplitudes. The gauge fixed action is local in spacetime if $S$ is local and if $\psi$ is chosen to be a local functional. This raises the question of whether the solution of the master equation is local in spacetime. [The measure terms in the path integral are of formal higher order in $\hbar$ and will not be considered here. A review of the antifield formalism may be found in [6].]

To analyze this question, one expands the solution $S$ of the master equation as a sum of terms of definite antighost number,

$$
S=\sum_{n \geqq 0} \stackrel{(n)}{S}, \quad \text { antigh } \stackrel{(n)}{S}=n, \quad \text { pure gh } \stackrel{(n)}{S}=n
$$

(0) $\stackrel{(1)}{S}$

The first terms $\stackrel{(0)}{S}$ and $\stackrel{(1)}{S}$ are given by $[1,6]$

$$
\stackrel{(0)}{S}=S_{0}
$$

where $S_{0}$ is the classical action and

$$
\stackrel{(1)}{S}=\phi_{i}^{*} R_{\alpha}^{i} C^{\alpha} .
$$

Here, $R_{\alpha}^{i}$ defines the gauge transformations,

$$
\delta_{\varepsilon} \phi^{i}=R_{\alpha}^{i} \varepsilon^{\alpha}
$$

while $C^{\alpha}$ and $\phi_{i}^{*}$ are respectively the ghosts and the antifields conjugate to $\phi^{i}$. 
The antifields conjugate to $C^{\alpha}$ are denoted by $C_{\alpha}^{*}$. One has

$$
\begin{aligned}
\text { pure gh } \phi^{i} & =\operatorname{antigh} \phi^{i}=0, \\
\text { pure gh } C^{\alpha} & =1, \quad \text { antigh } C^{\alpha}=0, \\
\text { pure gh } \phi_{i}^{*} & =0, \quad \text { antigh } \phi_{i}^{*}=1, \\
\text { pure gh } C_{\alpha}^{*} & =0, \quad \text { antigh } C_{\alpha}^{*}=2, \\
\text { gh } & =\text { pure gh }- \text { antigh. }
\end{aligned}
$$

The classical master equation

$$
(S, S)=0
$$

is equivalent to

$$
\delta \stackrel{(n+1)}{S}+\stackrel{(n)}{D}=0 \quad(n \geqq 1)
$$

where $\stackrel{(n)}{D}$ depends on $\stackrel{(k)}{S}$ with $k \leqq n$ and fulfills

$$
\delta \stackrel{(n)}{D}=0, \quad \text { antigh } \stackrel{(n)}{D}=n, \quad \text { pure } \operatorname{gh} \stackrel{(n)}{D}=n+1 .
$$

The Koszul-Tate differential $\delta$ in (6) and (7) is defined by

$$
\begin{gathered}
\delta \phi_{i}^{*}=\frac{\delta S_{0}}{\delta \phi^{i}}, \quad \delta C_{\alpha}^{*}=R_{\alpha}^{i} \phi_{i}^{*}, \\
\delta \phi^{i}=0, \quad \delta C^{\alpha}=0, \\
\text { antigh } \delta=-1, \quad \text { pure gh } \delta=0 .
\end{gathered}
$$

This derivation is nilpotent and has been shown in $[2,7,8]$, following standard methods of homological algebra, to be acyclic in antighost number $k>0$,

$$
H_{k}(\delta)=0, \quad k>0 .
$$

This implies that Eq. (6) possesses a solution for $\stackrel{(n+1)}{S}$. However, the problem of spacetime locality was not examined in $[2,7,8]$ and so, it is not clear from that analysis that (9) still holds in the space of local functionals. In other words, even though $\stackrel{(n)}{D}$ in (6) is a local functional as soon as the lower order terms $\stackrel{(k)}{S}$ are local functionals for $k \leqq n$, it is not clear that the solution $\stackrel{(n+1)}{S}$ of (6) is also a local functional.

The main result of this paper guarantees that this is so.

Theorem 1. Let A be a local functional,

with

$$
A=\int a\left(z^{A}, \partial_{\mu} z^{A}, \ldots, \partial_{\mu_{1}} \ldots \partial_{\mu_{S}} z^{A}\right) d x
$$

$$
\begin{gathered}
\text { antigh } A>0, \quad \text { pure gh } A>0 \\
z^{A} \equiv\left(\phi^{i}, C^{\alpha}, \phi_{i}^{*}, C_{\alpha}^{*}\right)
\end{gathered}
$$


If $\mathrm{A}$ is $\delta$-closed, then it is the $\delta$ of a local functional,

$$
\begin{gathered}
\delta A=0 \Rightarrow A=\delta B \\
B=\int b\left(z^{A}, \partial_{\mu} z^{A}, \ldots, \partial_{\mu_{1}} \cdots \partial_{\mu_{t}} z^{A}\right) d x .
\end{gathered}
$$

Differently said, the homology of $\delta$ is trivial

$$
H_{k}(\delta)=0, \quad k>0
$$

in the space of local functionals having both the antighost number and the pure ghost number strictly positive.

The proof of this theorem, announced in [6], is given in the subsequent sections.

The spacetime locality of each individual term $\stackrel{(n)}{S}$ in the expansion (1) implies the locality of the sum $S$ itself provided the theory is of finite rank, i.e., provided the sum (1) is finite. If the theory is of infinite rank, $S$ might in principle involve derivatives of the fields of arbitrarily high order. However, no irreducible gauge theory of infinite rank is known and so, it appears reasonable to make the assumption that the rank is finite. This will be done here.

The locality of $S$, in turn, implies the locality:

(i) of the BRST transformation, given by

$$
s z^{A}=\left(z^{A}, S\right)
$$

and (ii) of the gauge fixed action obtained by eliminating the antifields, provided one takes a gauge fixing fermion that is also a local functional.

The remaining part of this paper is devoted to the proof of Theorem 1 and is organized as follows.

In the next section, we reformulate the theorem in terms of the non-integrated densities $a$ and $b$ occurring in (10) and (11). We then explicitly state the technical assumptions on the action $S_{0}$ and the gauge transformations necessary to prove the theorem (Sect.4). One assumption concerns the regularity of the surface defined by the Euler-Lagrange equations $\delta S_{0} / \delta \phi^{i}=0$, the other is a local completeness condition on the gauge transformation. Finally, in Sect. 5, we prove the Theorem 1 or rather, its equivalent reformulation given in Sect.3. The last section contains concluding remarks.

\section{More on Local Functionals}

It is convenient to reformulate Theorem 1 in terms of the non-integrated densities $a$ and $b$ defining respectively $A$ and $B$. For that purpose, it is necessary to remove the integral sign. This can be done by means of the following theorem from the calculus of variations.

Theorem 2. If the variational derivatives

$$
\frac{\delta a}{\delta z^{A}} \equiv \frac{\partial a}{\partial z^{A}}-\partial_{\mu} \frac{\partial a}{\partial\left(\partial_{\mu} z^{A}\right)}+\partial_{\mu} \partial_{\nu} \frac{\partial a}{\partial\left(\partial_{\mu} \partial_{v} z^{A}\right)}-\cdots
$$


of a all identically vanish, then a is the sum of a total divergence and a constant,

$$
\frac{\delta a}{\delta z^{A}}=0 \Leftrightarrow a=\partial_{\mu} j^{\mu}+C .
$$

More generally, if $\delta a / \delta z^{1}=0$, then, $z^{1}$ and its derivatives can be removed from $a$ by adding a total divergence,

$$
\frac{\delta a}{\delta z^{1}}=0 \Leftrightarrow a=\bar{a}\left(z^{a}, \partial_{\mu} z^{a}, \ldots, \partial_{\mu_{1}} \cdots \partial_{\mu_{q}} z^{a}\right)+\partial_{\mu} j^{\mu} \quad(a=2,3, \ldots, n) .
$$

Proof: The proof of this theorem may be found in $[9,10,11]$, and more recently, in $[12,13]$.

It follows from the theorem that if $A=\int a d x=0$, then $a=\partial_{\mu} j^{\mu}$. Indeed, if $A=0$ for all field configuration, then, $\delta A / \delta z^{A}(x) \equiv \delta a / \delta z^{A}=0$ and so, $a=\partial_{\mu} j^{\mu}+C$. But the constant $C$ is easily seen to vanish as $A$ itself must be zero. ${ }^{1}$

Consequently, one can reformulate Theorem 1 as follows.

Theorem 3. Let a $\left(z^{A}, \ldots, \partial_{\mu_{1}} \cdots \partial_{\mu_{s}} z^{A}\right)$ be a non-integrated density with

$$
\text { antigh } a>0, \quad \text { pure gh } a>0 \text {. }
$$

If a is $\delta$-closed modulo a total divergence,

$$
\delta a=\partial_{\lambda} \mu^{\lambda}
$$

then, it is automatically $\delta$-exact modulo a total divergence,

$$
a=\delta b+\partial_{\lambda} v^{\lambda}
$$

$(16 a) \Rightarrow(16 b)$.

The homology of $\delta$ modulo the exterior spacetime derivative is thus trivial at positive antighost and pure ghost numbers. [This latter statement uses dual terminology, in which the function $a$ is replaced by the dual $D$-form $a d x^{0} \wedge \cdots \wedge d x^{D-1}$, and $\partial_{\lambda} \mu^{\lambda}$ is replaced by $d \mu$, where $\mu$ is the $(D-1)$-form dual to $\mu^{\lambda}$.]

It is this equivalent version of Theorem 1 that will be proved in the sequel.

For that purpose, the following theorem will be needed

Theorem 4. If $\partial_{\mu} j^{\mu}=0$, then $j^{\mu}=\partial_{v} S^{\mu v}+C^{\mu}$, where $S^{\mu v}=-S^{v \mu}$ and $C^{\mu}=$ const.,

$$
\partial_{\mu} j^{\mu}=0 \text { (identically) } \Leftrightarrow j^{\mu}=\partial_{v} S^{v \mu}+C^{\mu}, S^{v \mu}=-S^{v \mu} \text {. }
$$

Proof. Again, this a well-known theorem from the calculus of variations. A recent proof is given in $[12,13]$. For completeness, another proof is given in the appendix.

\section{Assumptions on the Action $S_{0}$ and the Gauge Transformations}

4.1. Regularity Conditions. The action is a local functional,

$$
S_{0}\left[\phi^{i}\right]=\int \mathscr{L}_{0} d x
$$

\footnotetext{
${ }^{1}$ As a rule, it will always be assumed that the boundary conditions are such that the flux of the currents that appear are zero
} 
and the Euler-Lagrange equations

$$
\frac{\delta \mathscr{L}_{0}}{\delta \phi^{i}}=0
$$

are therefore partial differential equations of finite order for $\phi^{i}$.

Let $V^{n}$ be the space of the fields $\phi^{i}$ and their successive derivatives $\partial_{\mu_{1}} \ldots \partial_{\mu_{k}} \phi^{i}$ up to order $n$ at some arbitrary but unspecified spacetime point. For an arbitrary field configurations, these variables are independent (they are only subject to the symmetry properties $\partial_{\mu} \partial_{\nu} \phi^{i}=\partial_{\nu} \partial_{\mu} \phi^{i}$ etc...). Let $s$ be the order of the highest derivative occurring in $\delta \mathscr{L}_{0} / \delta \phi^{i}$,

$$
\frac{\delta \mathscr{L}_{0}}{\delta \phi^{i}} \equiv \frac{\delta \mathscr{L}_{0}}{\delta \phi^{i}}\left(\phi^{j}, \partial_{\mu} \phi^{j}, \ldots, \partial_{\mu_{1}} \ldots \partial_{\mu_{s}} \phi^{i}\right)
$$

The equations

$$
\frac{\delta \mathscr{L}_{0}}{\delta \phi^{i}}=0
$$

define a surface in $V^{s}$. Similarly, the equations

$$
\frac{\delta \mathscr{L}_{0}}{\delta \phi^{i}}=0, \quad \partial_{\mu} \frac{\delta \mathscr{L}_{0}}{\delta \phi^{i}}=0, \ldots, \partial_{\mu_{1}} \ldots \partial_{\mu_{k}} \frac{\delta \mathscr{L}_{0}}{\delta \phi^{i}}=0
$$

define a surface in $V^{s+k}$.

We will assume that for any $k$, the equations (20b) indeed define a smooth surface and provide a "regular" representation of that surface.

[One says that the equations $G_{a}=0$ provide a regular representation of a surface $\sum$ if one can locally split the functions $G_{a}$ as $G_{a} \equiv\left(G_{A}, G_{\alpha}\right)$ in such a way that (i) $G_{\alpha}=0$ is a consequence of $G_{A}=0$; and (ii) the exterior form $\wedge_{A} d G_{A}$ does not vanish on $\sum\left(0\right.$ is a regular value of the map defined by $\left.G_{A}\right)$.]

The regularity condition is usually fulfilled, at least almost everywhere, by most of the theories of physical interest. If there are constraints on the dynamical variables enforced in the variational principle by means of Lagrange multipliers, the regularity condition forbids one to replace them by, say, their squares [14].

In principle, one should check that the regularity condition holds for any value of $k$. In practice, however, the verification is finished as soon as the independent equations among (20b) form a "passive" partial differential system in the sense of [15].

4.2 Local Completeness of the Gauge Transformations. The symbolic form (3) of the gauge transformations actually stands for

$$
\delta_{\varepsilon} \phi^{i}=r_{\alpha}^{i} \varepsilon^{\alpha}+r_{\alpha}^{i \mu} \partial_{\mu} \varepsilon^{\alpha}+\cdots+r_{\alpha}^{i \mu_{1} \ldots \mu_{t}} \partial_{\mu_{1}} \ldots \partial_{\mu_{t}} \varepsilon^{\alpha},
$$

where the functions $r_{\alpha}^{i}, r_{\alpha}^{i \mu}, \ldots$ and $r_{\alpha}^{i \mu_{1} \ldots \mu_{t}}$ depend on the fields on their derivatives up to some finite order, and where the $\varepsilon^{\alpha}$ are arbitrary functions of $x$. From the invariance of the action under (21), one gets the Noether identities

$$
\frac{\delta \mathscr{L}_{0}}{\delta \phi^{i}} r_{\alpha}^{i}-\partial_{\mu}\left(\frac{\delta \mathscr{L}_{0}}{\delta \phi^{i}} r_{\alpha}^{i \mu}\right)+\cdots+(-)^{t} \partial_{\mu_{1}} \ldots \partial_{\mu_{t}}\left(\frac{\delta \mathscr{L}_{0}}{\delta \phi^{i}} r_{\alpha}^{i \mu_{1} \ldots \mu_{t}}\right)=0
$$


We will assume that the gauge transformations (21) are not only complete in the sense of [6], but also locally complete. By this it is meant:

(i) ("completeness") that any gauge transformation can be expressed in terms of (21) up to gauge transformations vanishing on-shell.

(ii) ("local completeness") that any gauge transformation given by a local expression can be expressed locally in terms of (21); or, what is the same, that any local identity on $\delta \mathscr{L}_{0} / \delta \phi^{i}$ and its derivatives can be derived from (22) by local means, i.e., by algebraic manipulations and differentiations but no integration. So, if the equation

$$
\int T^{i}\left(x, x^{\prime}\right) \frac{\delta S_{0}}{\delta \phi^{i}(x)} d x=0
$$

holds identically, where $T^{i}\left(x, x^{\prime}\right)$ involves the delta function $\delta\left(x, x^{\prime}\right)$ and its derivatives up to some finite order, then it must be true that

$$
\begin{aligned}
T^{i}\left(x, x^{\prime}\right)= & \int d x^{\prime \prime} R_{\alpha}^{i}\left(x, x^{\prime \prime}\right) \mu^{\alpha}\left(x^{\prime \prime}, x^{\prime}\right) \\
& +\int d x^{\prime \prime} v^{i j}\left(x, x^{\prime}, x^{\prime \prime}\right) \frac{\delta S_{0}}{\delta \phi^{j}\left(x^{\prime \prime}\right)}, \\
v^{i j}\left(x, x^{\prime}, x^{\prime \prime}\right)= & -v^{j i}\left(x^{\prime \prime}, x^{\prime}, x\right),
\end{aligned}
$$

where both $\mu^{\alpha}\left(x^{\prime \prime}, x^{\prime}\right)$ and $v^{i j}\left(x^{\prime \prime}, x^{\prime}, x\right)$ involve the delta functions $\delta\left(x^{\prime \prime}, x^{\prime}\right), \delta\left(x^{\prime}, x\right)$ and their derivatives up to a finite order, but no primitive of $\delta\left(x, x^{\prime}\right)$. [Of course, these functions also possess an unwritten dependence on the fields and their derivatives.]

For instance, in the case of the Maxwell theory, the gauge transformations are

$$
\delta_{\varepsilon} A_{\mu}(x)=\partial_{\mu} \varepsilon(x)=\int d x^{\prime} \frac{\partial}{\partial x^{\mu}} \delta\left(x, x^{\prime}\right) \varepsilon\left(x^{\prime}\right)
$$

and yield the Noether identity

$$
\partial_{\mu} \frac{\delta \mathscr{L}_{0}}{\delta A_{\mu}}=0
$$

If one replaces $\varepsilon$ by $\square \eta$ in (23a), one gets a different form of the gauge transformations,

$$
\delta_{\eta} A_{\mu}(x)=\partial_{\mu} \square \eta(x)=\int d x^{\prime} \square \frac{\partial}{\partial x^{\mu}} \delta\left(x, x^{\prime}\right) \eta\left(x^{\prime}\right),
$$

which leads to

$$
\square \partial_{\mu} \frac{\delta \mathscr{L}_{0}}{\delta A_{\mu}}=0
$$

This second form of the gauge transformations is not locally complete because one cannot express the coefficient $\delta_{, \mu}\left(x, x^{\prime}\right)$ appearing in (23a) in terms of $\square \delta_{, \mu}\left(x, x^{\prime}\right)$ by mere differentiation: one must invert $\square$, and this is a non-local question. By contrast, the representation (23a) of the gauge symmetry is locally complete.

By redefining the gauge transformations, or by completing the given set of 
gauge transformations (increasing possibly the reducibility), one can assume that the local completeness condition is fulfilled.

In a locally complete representation of the gauge symmetry, the commutator of two gauge transformations can be expressed locally in terms of the original gauge transformations and the equations of motion.

\section{Homology of $\delta$ Modulo $d$}

Under the above assumptions, the proof of Theorem 3 is direct.

5.1 Local Cohomology of $\delta$. The Koszul-Tate differential (8) explicitly reads, in uncondensed notations

$$
\begin{gathered}
\delta \phi_{i}^{*}=\frac{\delta \mathscr{L}_{0}}{\delta \phi^{i}}, \\
\delta C_{\alpha}^{*}=r_{\alpha}^{i} \phi_{i}^{*}-\partial_{\mu}\left(r_{\alpha}^{i \mu} \phi_{i}^{*}\right)+\cdots+(-)^{t} \partial_{\mu_{1}} \ldots \partial_{\mu_{t}}\left(r_{\alpha}^{i \mu_{1} \cdots \mu_{t}} \phi_{i}^{*}\right), \\
\delta \phi^{i}=0 \quad \delta C^{\alpha}=0 .
\end{gathered}
$$

Its action on the derivatives of the $z$ 's is obtained by using the rule

$$
\delta \partial_{\mu}=\partial_{\mu} \delta
$$

The Koszul-Tate differential implements the restriction to the surface $\delta \mathscr{L}_{0} /$ $\delta \phi^{i}=0, \partial_{\mu} \delta \mathscr{L}_{0} / \delta \phi^{i}=0, \ldots$ Each of the equations $\delta \mathscr{L}_{0} / \delta \phi^{i}=0, \partial_{\mu} \delta \mathscr{L}_{0} / \delta \phi^{i}=$ $0, \ldots$ has an independent antifield, namely, $\phi_{i}^{*}, \partial_{\mu} \phi_{i}^{*}, \ldots$ Furthermore, for each independent identity among the equations, there is an independent "antifield of antifield," which is either $C_{\alpha}^{*}$ or one of its appropriate derivatives.

Accordingly, by using standard methods of homological algebra, one can show that any $\delta$-closed function of the fields and their derivatives which has positive antighost number is also $\delta$-exact,

$$
\delta a=0, \quad \text { antigh } a>0 \Rightarrow a=\delta b,
$$

i.e.,

$$
H_{k}^{\text {loc }}(\delta)=0, \quad k>0 .
$$

To that end, one constructs a contracting homotopy $\sigma$,

$$
\sigma \delta+\delta \sigma=N
$$

where $N$ counts the number of antifields, of equations $\delta \mathscr{L}_{0} / \delta \phi^{i}$ and of their derivatives contained in a (see e.g. [7] for more information).

Even though $\delta$ commutes with $\partial_{\mu}$, the contracting homotopy $\sigma$ cannot be chosen, in general, to commute with $\partial_{\mu}$. This would imply that the homology of $\delta$ modulo $d$ is trivial at positive antighost number $\left(\delta a=\partial_{\mu} j^{\mu} \Rightarrow a=\delta\left(\frac{1}{n} \sigma a\right)+\right.$ $\left.\partial_{\mu}\left(\frac{1}{n} \sigma j^{\mu}\right)\right)$, and this may not be the case.

That the homology of $\delta$ modulo $d$ may be non-trivial at positive antighost 
number can be seen on the example $\mathscr{L}_{0}=\dot{q}^{2} / 2$. One has $\delta q^{*}=\ddot{q}=d \dot{q} / d t$ and yet $q^{*} \neq \delta \alpha+d \beta / d t$. [If $q^{*}=\delta \alpha+d \beta / d t$, then also $q^{*}=d \bar{\beta} / d t$ with $\bar{\beta}=\beta(\ddot{q}=0$, $\ddot{q}=0, \ldots)$. But this is impossible because the variational derivatives of $q^{*}$ do not vanish.]

This is the reason why the extra condition on the pure ghost number is imposed in Theorem 3 (and Theorem 1).

Because $\delta$ does not act at all on the ghosts $C^{\alpha}$, one can, however, choose $\sigma$ to commute with $\bar{\partial}_{\mu}$, where $\bar{\partial}_{\mu}$ is the operator that differentiates only with respect to the ghosts,

$$
\begin{gathered}
\bar{\partial}_{\mu}=C_{, \mu}^{\alpha} \frac{\partial}{\partial C^{\alpha}}+C_{, \mu \nu}^{\alpha} \frac{\partial}{\partial C_{, \mu}^{\alpha}}+\cdots, \\
\sigma \bar{\partial}_{\mu}=\bar{\partial}_{\mu} \sigma .
\end{gathered}
$$

The complete derivative $\partial_{\mu}$ is the sum

$$
\partial_{\mu}=\tilde{\partial}_{\mu}+\bar{\partial}_{\mu}
$$

where $\tilde{\partial}_{\mu}$ acts on all the variables but the ghosts. The homology of $\delta$ modulo $\bar{\partial}_{\mu}$ is therefore trivial,

$$
\delta a=\bar{\partial}_{\mu} j^{\mu}, \quad \text { antigh } a>0 \Rightarrow a=\delta b+\bar{\partial}_{\mu} \lambda^{\mu} .
$$

5.2 Proof of Theorem 3. The proof of Theorem 3 crucially relies on (28) and follows a perturbative argument similar to spectral sequence techniques.

Let $M$ be the even differential that counts the number of derivatives of the ghosts,

$$
\begin{gathered}
M \partial_{\mu_{1}} \ldots \partial_{\mu_{k}} \phi^{i}=0, \quad M \partial_{\mu_{1}} \ldots \partial_{\mu_{k}} \phi_{i}^{*}=0, \quad M \partial_{\mu_{1}} \ldots \partial_{\mu_{k}} C_{\alpha}^{*}=0 \\
M \partial_{\mu_{1}} \ldots \partial_{\mu_{k}} C^{\alpha}=k \partial_{\mu_{1}} \ldots \partial_{\mu_{k}} C^{\alpha} .
\end{gathered}
$$

A similar differential was introduced in $[12,16]$ in the study of the BRST cohomology for Yang-Mills models. One has

$$
\left[M, \tilde{\partial}_{\mu}\right]=0, \quad\left[M, \bar{\partial}_{\mu}\right]=\bar{\partial}_{\mu} .
$$

Let $a$ be $\delta$-closed modulo $\partial_{\lambda} \mu^{\lambda}$, with antigh $a>0$, pure gh $a>0$. The local functions $a$ and $\mu^{\lambda}$ can be expanded according to the number of derivatives of the ghosts,

$$
\begin{gathered}
a=\stackrel{(0)}{a}+\cdots+\stackrel{(s)}{a}, \\
\mu^{\lambda}=\stackrel{(0)}{\mu^{\lambda}}+\cdots+\stackrel{(t)}{\mu^{\lambda},}, \\
M \stackrel{(k)}{a}=k \stackrel{(k)}{a}, \quad M^{(k)} \mu^{\lambda}=k \stackrel{(k)}{\mu^{\lambda}} .
\end{gathered}
$$

The expansions (31) are finite, because $a$ and $\mu^{\lambda}$ are polynomials in the ghosts (they possess definite ghost number) and are local, i.e., contain the derivatives $\partial_{\mu_{1}} \ldots \partial_{\mu_{q}} C^{\alpha}$ up to a finite order.

The equation $\delta a=\partial_{\lambda} \mu^{\lambda}$ is equivalent to

$$
\delta \stackrel{(i)}{a}=\bar{\partial}_{\lambda}{ }^{(i-1) \lambda} \mu+\tilde{\partial}_{\lambda}{ }^{(i) \lambda} \mu \text {. }
$$


Without loss of generality, one can assume $t=s-1$, since otherwise Eq. (32) with $i=t+1$ reads

$$
\bar{\partial}_{\lambda}^{(t) \lambda} \mu=0
$$

which implies

$$
\stackrel{(t) \lambda}{\mu}=\bar{\partial}_{v} S^{\lambda \nu}, \quad S^{\lambda v}=-S^{\nu \lambda}, \quad M S^{\lambda v}=(t-1) S^{\lambda v}
$$

(i) $\lambda$

(Theorem 4). The ghost-independent term in (17) disappears because pure gh $\mu>0$. But then, $\mu^{, \lambda}=\mu^{\lambda}-\partial_{\nu} S^{\lambda \nu}$ is also such that $\delta a=\partial_{\lambda} \mu^{, \lambda}$ and contains only terms of degree $\leqq t-1$. So, one can assume $t=s-1$.

With $t=s-1$, Eq. (32) for $i=s$ is given explicitly by

$$
\delta \stackrel{(s)}{a}=\bar{\partial}_{\lambda} \stackrel{(s-1) \lambda}{\mu} .
$$

Using the fact that the $\delta$-homology modulo $\bar{\partial}_{\lambda}$ is trivial, one gets

$$
\stackrel{(s)}{a}=\delta \stackrel{(s)}{b}+\bar{\partial}_{\lambda} \stackrel{(s-1) \lambda}{v}
$$

and so, the function $a-\delta \stackrel{(s)}{b}-\partial_{\lambda} \stackrel{(s-1) \lambda}{v}=a-\stackrel{(s)}{a}-\tilde{\partial}_{\lambda}{ }^{(s-1) \lambda} v$ is $\delta$-closed modulo $d$ and has no component of $M$-degree $s$. Going on in the same fashion, one then removes successively the remaining components of $M$-degrees $s-1, s-2, \ldots$ by adding an appropriate $\delta$-exact term and a total divergence. Collecting the terms, one ultimately gets

$$
a=\delta b+\partial_{\lambda} v^{\lambda}
$$

which is the desired result. This completes the proof of Theorem 3.

\section{Concluding Remarks}

(i) One can analyse in a similar fashion the homology of $\delta$ modulo the exterior spacetime $d$ for other values of the form-degree. One finds again that the homology is trivial when both the antighost number and the pure ghost number are strictly positive.

(ii) If the antighost number of $a$ is strictly greater than the spacetime dimension $D$, one can provide a different proof of the triviality of the homology of $\delta$ modulo $d$. This alternative proof does not require pure gh $a>0$ but fails if antigh $a \leqq D$. It is based on "zig-zag equations" in the double complex based on $\delta$ and $d$ and goes as follows.

The equation

$$
\delta \stackrel{(D)}{a}=d^{(D-1)} \mu,
$$

where the superscript denotes the form-degree (we revert to dual notations), implies successfully

$$
\begin{gathered}
\delta^{(D-1)}=d^{(D-2)} \mu, \\
\delta^{(D-2)}=d^{(D-3)} \mu, \\
\vdots \\
\delta^{(0)} \mu=0 .
\end{gathered}
$$


This follows from the triviality of the $d$-cohomology (Theorem 4): the constant terms drop out because the $\mu$ 's have strictly positive antighost number. Furthermore, antigh $\stackrel{(0)}{\mu}=$ antigh $a-D \geqq 1$. Hence, $\stackrel{(0)}{\mu}$ is $\delta$-exact, $\stackrel{(0)}{\mu}=\delta \stackrel{(0)}{v}$. By going up the ladder, one then gets $\stackrel{(1)}{\mu}+d \stackrel{(0)}{v}=\delta \stackrel{(1)}{v}, \ldots$, and finally, $\stackrel{(D)}{a}+d \stackrel{(D-1)}{v}=\delta \stackrel{(D)}{v}$ as required.

(iii) The triviality of the homology of $\delta$ modulo $d$ for antigh $>0$ and pure gh $>0$ can be used to prove that the BRST invariant extension of any on-shell gauge invariant local functional (more generally, any local, closed, longitudinal form along the gauge orbits) is also a local functional.

(iv) A different approach may be found in [17]

Acknowledgements. The author is grateful to M. Dubois-Violette, J. Fisch, M. Talon and C. Viallet for useful conversations.

\section{Appendix}

We prove in this appendix Theorem 4: if the divergence $\partial_{\mu} j^{\mu}=0$ vanishes identically, then $j^{\mu}$ is the sum of the divergence of an antisymmetric tensor and a constant current, $j^{\mu}=\partial_{v} S^{\mu \nu}+C^{\mu}$ with $S^{\mu v}=-S^{v \mu}$. Here, the $C^{\mu}$ are constant.

First, one needs the following lemma.

Lemma. Let $y^{\alpha} \equiv\left(y^{a}, y^{N+1}\right), a=1, \ldots, N, \alpha=1, \ldots, N+1$, be $N+1$ independent fields and let $\mathrm{m}$ be a local function of the first $\mathrm{N}$ fields $\mathrm{y}^{a}$ and their derivatives. If

$$
\sum_{a=1}^{N} \frac{\delta m}{\delta y^{a}} y^{a+1}=\partial_{\mu} \beta^{\mu},
$$

then

$$
\frac{\delta m}{\delta y^{a}}=0
$$

i.e.,

$$
m=\partial_{\mu} n^{\mu}+C .
$$

The proof is straightforward: because the left-hand side of (A.1) depends only linearly on $y^{N+1}$ and does not contain the derivatives of $y^{N+1}$, it must actually be independent of $y^{N+1}$, i.e., $\delta m / \delta y^{N}=0$ (the right-hand side of (A.1) necessarily contains derivatives of $y^{N+1}$ if it contains $\left.y^{N+1}\right)$. Repeating the argument, one then finds (A.3).

Once the lemma is established, the proof of Theorem 4 is direct and proceeds by induction on the dimension.

(i) The theorem is easily seen to hold in one spacetime dimension.

(ii) If true in $n$ spacetime dimensions (for any number of fields), the theorem is then also true in $n+1$ dimensions.

Indeed, straightforward integrations by parts in

$$
\partial_{0} m^{0}+\partial_{k} m^{k}=0 \quad(k=1, \ldots, n)
$$


yield

$$
\frac{\delta m^{0}}{\delta z^{A}} \frac{\partial z^{A}}{\partial x^{0}}+\frac{\delta m^{(0)}}{\partial\left(\partial_{0} z^{A}\right)} \frac{\partial^{2} z^{A}}{\left(\partial x^{0}\right)^{2}}+\cdots+\frac{\delta m^{(0)}}{\delta\left(\partial^{u} z^{A} /\left(\partial x^{0}\right)^{u}\right)} \frac{\partial^{u+1} z^{A}}{\left(\partial x^{0}\right)^{u+1}}=\partial_{k} \bar{m}^{k}
$$

Here, the variational derivatives are those in $n$ dimensions, for which $z^{A}, \partial_{0} z^{A}$, $\partial_{00} z^{A}, \ldots$ are independent. An application of the lemma then gives

$$
m^{0}=\partial_{k} S^{0 k}+C^{0}
$$

which, when substituted in $\partial_{\mu} m^{\mu}=0$, implies

$$
m^{k}=\partial_{0} S^{k 0}+\partial_{m} S^{k m}+C^{k}
$$

since the theorem is true in $n$ dimensions. Here

$$
S^{k 0}=-S^{k 0}
$$

and

$$
S^{k m}=-S^{m k}
$$

This proves the theorem.

Remarks. (i) The Theorem 4 shows that the cohomology in degree D-1 of the algebraic $d$ defined in the space of functions of the fields and their derivatives by

$$
d m=\left(\partial_{\mu} m\right) d x^{\mu}=\left[\frac{\partial m}{\partial z^{A}} \partial_{\mu} z^{A}+\cdots+\frac{\partial m}{\partial\left(\partial_{\mu_{1}} \ldots \partial_{\mu_{s}} z^{A}\right)} \partial_{\mu_{1}} \ldots \partial_{\mu_{s}} \partial_{\mu} z^{A}\right] d x^{\mu}
$$

is exhausted by the constants. Here, $D$ is the spacetime dimension. A similar property actually holds for any degree $<D$ and is proven along identical lines.

(ii) If $\partial_{\mu}$ in Theorem 4 does not act on some variables, then the constant current $C^{\mu}$ can depend on all the variables not "seen" by $\partial_{\mu}$.

\section{References}

1. Batalin, I. A., Vilkovisky, G. A.: Phys. Lett. 102B, 27 (1981); 120B, 166 (1983); Phys. Rev. D28 2567 (1983). Some features of the antifield formalism were anticipated In: Kallosh, R. E.: Nucl. Phys. 141, 141 (1978); de Wit, B., van Holten J.W.: Phys. Lett. 79B, 389 (1979)

2. Fisch, J. M. L., Henneaux, M.: Commun. Math. Phys. 128, 627 (1990)

3. Batalin, I. A., Fradkin, E. S.: Rev. Nuovo Cimento 9, 1 (1986)

4. Henneaux, M.: Phys. Rep. 126, 1 (1985)

5. Voronov, B. L., Tyutin, I. V.: Theor. Math. Phys. 50, 218 (1982)

6. Henneaux, M.: Lectures on the Antifield-BRST Formalism for Gauge Theories,' Lectures given in June 1989, Nucl. Phys. B (Proc. Suppl.) 18A, 47 (1990); to appear also in "Quantum Mechanics of Fundamental Systems III, C. Teitelboim and J. Zanelli (eds.). New York: Plenum Press

7. Fisch, J., Henneaux, M., Stasheff, J., Teitelboim, C.: Commun. Math. Phys. 120, 379 (1989)

8. Batalin I. A. and Vilkovisky, G.A.: J. Math. Phys. 26, 172 (1985)

9. Koenigsberger, L.: J. für Reine u. Angewandte Mathematik, Heft 3, Bd CXXIV (1910)

10. Henneaux, M.: Bull. Cl. Sc. Acad. Roy. Belg. LXIV (1978) 440 (the proof given there is valid for functions depending on the fields and their first derivatives only)

11. Dixon, J.A.: Cohomology and renormalization of gauge fields I, II, III and IV, „ unpublished 
12. Dixon, J.A.: Spectral Sequence Calculation of the restricted local BRST Cohomology... University of Victoria (BC) preprint (1989)

13. Brandt, F., Dragon, N., Kreuzer, M.: DESY preprint 89-089 (1989); see also Brandt, F. Dragon, N., Kreuzer, M.: Phys. Lett. 213B, 263 (1989)

14. Dirac, P.A.M.: Can. J. Math. 2, 129 (1950)

15. Riquier, C.: Les Systèmes d'Equations aux Dérivées Partielles, Paris 1910; Janet, M.: Leçons sur les Systèmes d'Equations aux Dérivées Partielles, Paris 1929; Douglas, J.: Trans. Am. Math. Soc. 50, 71 (1941)

16. Bandelloni, G.: Nuovo Cimenta 88, (1) 35 (1985); J. Math. Phys. 27, 1128 (1986)

17. Dubois-Violette, M., Henneaux, M., Talon, M., Viallet, C. M.: Paris VI preprint PAR-LPTHE 91/19 (1991)

Communicated by K. Gawedzki 
